\title{
Study on rickettsial diseases evidenced by Weil-Felix test among febrile patients visiting a tertiary care hospital in Mymensingh
}

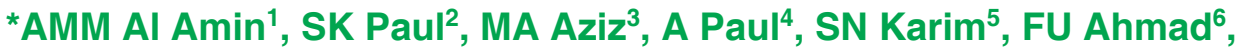 \\ M Alam ${ }^{7}$, R Mazid ${ }^{8}$, MA Hossain ${ }^{9}$
}

\begin{abstract}
Background: Rickettsial diseases are neglected, re-emerging vector borne zoonosis \& increasingly considered as one of the most important causes of pyrexia of unknown origin (PUO). They are grossly underdiagnosed due to wide range of non-specific symptoms, low index of clinical suspicion, lack of widely available specific diagnostic tools, leading to significant morbidity \& mortality. Appropriate diagnosis in early stages is therefore necessary to prevent fatal complications associated with this disease. Objective: Present study was attempted to assess the burden of rickettsial illness by Weil-Felix agglutination test, among the suspected febrile patients, visiting a tertiary care hospital as well as to analyze the demographic profile \& clinical manifestations of the seropositive cases. Methods: This was a cross-sectional study, conducted at department of microbiology, Mymensingh Medical College from March 2018 to February 2019. A total of 453 febrile patients of suspected rickettsial illness, irrespective of age and sex, were enrolled in this study. Serum sample from all the enrolled cases were then analyzed for rickettsial antibodies by Weil-Felix slide agglutination test. Results: Out of 453 cases, a total of 260 (57.39\%) showed significant agglutination by Weil-Felix test, of which 101 (38.84\%) were reactive to OX2 (spotted fever group rickettsiae), 65 (25\%) were reactive to OXK (Scrub typhus) \& $13(5 \%)$ showed significant titers to OX19 (typhus fever). Remaining 81 $(31.15 \%)$ sera were reactive to more than one antigens (mixed reactivity). Seropositivity was higher among female subjects $(142 ; 54.61 \%)$ \& age group $>15-30$ accounted for highest number of cases $(95 ; 36.53 \%)$. Positive cases showed diverse clinical manifestations like headache $(55.76 \%)$, myalgia $(50.76 \%)$, skin rash $(10.38 \%)$, eschar $(9.23 \%)$, oliguria $(7.3 \%)$, jaundice $(10.76 \%)$, splenomegaly $(6.81 \%)$, hepatomegaly $(7.30 \%)$ etc. Conclusion: Rickettsial diseases should be considered as an important etiology of PUO \& early diagnosis should be done to initiate proper treatment to prevent fatal complications. Though it lacks sensitivity \& specificity, in a resource constraint area like Bangladesh, Weil-Felix test still serves as the cheapest initial diagnostic tool for rickettsial illness to guide the physician for further approaches.
\end{abstract}

1. Dr. Abu Md. Mayeenuddin Al Amin, Assistant professor, Department of Microbiology, Gazi Medical College, Khulna. mayeenuddinamin@gmail.com

2. Professor Dr. Shyamal Kumar Paul, Professor of Virology \& Principal, Netrokona Medical College, Netrokona.

3. Dr. Md. Abdul Aziz, Assistant professor (c.c.), Department of Microbiology, Rangpur Medical College, Rangpur.

4. Dr. Anindita Paul, Lecturer, Department of Microbiology, Mymensingh Medical College, Mymensingh.

5. Dr. Syeda Noorjahan Karim, Assistant professor, Department of Pathology, Gazi Medical College, Khulna.

6. Dr. Fahim Uddin Ahmad, Assistant professor, Department of Microbiology, TMSS Medical College, Bogura.

7. Dr. Mahbubul Alam, Lecturer, Department of Microbiology, Shaheed Tajuddin Ahmed Medical College, Gazipur.

8. Dr. Rummana Mazid, Consultant, Clinical Microbiology Laboratory, BIHS General Hospital, Dhaka.

9. Professor Dr. Md. Akram Hossain, Senior Consultant, Microbiology, Imperial Hospital Limited, Chattogram. 


\section{Introduction}

Rickettsiae are a group of small gram negative, non-motile, obligate intracellular organisms, those are transmitted directly to human hosts by the bite of arthropod vectors likeLouse, mite, fleas and ticks. ${ }^{1}$ They are actually pleomorphic coccobacilli, not well stained with Gram's technique but can be seen under light microscope, when Giemsa or acridine orange staining are applied. ${ }^{2}$ Rickettsial diseases are considered as a re-emerging threat to public health, if not early diagnosed or misdiagnosed. Various type of rickettsial infections are now enlisted as an important cause of pyrexia of unknown origin (PUO) and therefore it is required to be differentiated from many other common febrile illnesses like- dengue, malaria, enteric fever, leptospirosis, etc. ${ }^{3}$ Rickettsial agents are categorized into 3 main biogroups. Typhus fever group comprising of R. prowazekii \& R. typhi, which are responsible for classical epidemic typhus \& endemic typhus respectively. Spotted fever group contain many species like- $\mathrm{R}$. rickettsia, R. conorii, causing Rocky mountain spotted fever. Scrub typhus is another entity which is caused by a closely related rickettsial agentOrientia tsutsugamushi, which is transmitted to human hosts by larval trombiculid mites. ${ }^{4}$ Rickettsial diseases are regarded as one of the main causes of nonviral illnesses, that present with fever \& rash. ${ }^{5}$ Severities of this disease may vary from self-limiting febrile illness to a fulminant life threatening infection including multiorgan dysfunction with reported case fatality ranging from $1 \%$ to $30 \%$, if left untreated. ${ }^{6}$ After an incubation period of 3-14 days, there is a sudden onset of non-specific symptoms like- fever, chill, headache, myalgia, dry cough, nausea, vomiting, lymphadenopathy and often a maculopapular rash on the extremeties. ${ }^{7}$ An Eschar is a pathognomonic sign for scrub typhus, which is a cigarette-burn like cutaneous black necrotic lesion, appears on bite site of the larval mite, variably seen in $50 \%$ of cases. ${ }^{8}$
However, occurrence of Escher is rare in south east Asian patients. ${ }^{4}$ Recent studies in Asian countries have reported unusual complications of rickettsial diseases, those usually develop after first week of illness likegastrointestinal manifestation, renal failure, hepatitis, pneumonitis, acute respiratory distress syndrome (ARDS), septic shock, myocarditis, meningoencephalitis, tinnitus and hearing loss. ${ }^{9,10}$

Rickettsial illnesses are frequently misdiagnosed by physicians due to lack of data regarding geographical distribution, low index of suspicion, non-specific clinical manifestations \& unavailability of sensitive as well as specific diagnostic tests, leading to significant morbidity \& mortality. ${ }^{2,11}$ Serological methods like- Weil-Felix test (WFT), latex agglutination test, immunoperoxidase assay, Enzyme Linked Immunosorbent Assay (ELISA), and micro immunofluorescence ("gold standard"), can be used for diagnosing suspected rickettsial infections. The most widely used method is Weil-Felix test which is a non-specific heterophile agglutination test. It was developed from observation of Weil \& Felix that, sera from patients with typhus fever agglutinated certain strains of Proteus like- Proteus vulgaris OX-19 and OX-2 as well as Proteus mirabilis OX-K. The test is usually done as tube agglutination though, rapid slide agglutination methods have been employed for screening. ${ }^{12}$ Though this test lacks both sensitivity and specificity, in most of the laboratories in resource limiting area, this WF test is the only one available method. ${ }^{1}$

Rickettsial infections prevail world-wide and they have been reported from all parts of India like Jammu, Kashmir, Himachal Pradesh, Rajasthan, Assam, Kerala, Uttar-Pradesh, Tamil-Nadu, West Bengal, Maharashtra.13 Recently, rickettsial illnesses like- Scrub typhus and murine typhus also have been reported from Chittagong, Bangladesh.14 But the data regarding the burden of rickettsial infections in our country is very limited, which actually reflects only the tip of the iceberg. 


\section{Methodology}

This was a cross-sectional study, conducted at department of microbiology, Mymensingh Medical College from the period of March 2018 to February 2019. A total of 453 Febrile patients (fever for more than 5 days) of suspected rickettsial illness, irrespective of age and sex, referred from outpatient and inpatient facilities of department of Medicine and department of Pediatrics, Mymensingh Medical College Hospital $(\mathrm{MMCH})$ were included in the study. Febrile patients with already established cause of their illness other than rickettsial disease, were excluded from this study.

Before collecting blood sample, informed consent was taken from all patients \& socio-demographic data of all the subjects were collected by using pre-tested, printed questionnaire. Following all universal safety precautions, $2 \mathrm{ml}$ of venous blood was collected with a sterile disposable syringe \& was transferred into a clean sterile test tube \& blood was kept for 30 minutes for clot formation. Following centrifugation of blood at 800 rpm for 10 minutes, serum was separated from the clotted blood \& then collected into a sterile microcentrifuge tube for Weil-Felix test. The serum samples were tested immediately. Whenever a delay in testing was anticipated, serum samples were stored in $-20^{\circ} \mathrm{C}$. According to the manufacturer's instructions, Weil-Felix slide agglutination assay were performed as a rapid screening test, on each serum sample, using all the three proteus strains- P. vulgaris OX-19, OX-2 and p. mirabilis OX-K (Cypress Diagnostics, Schotelveldstraat 3B-3012, Leuven, Belgium). First, the smooth stained Cypress antigen suspensions were mixed with patient's serum. Agglutination obtained within 1 minute was considered as positive reaction, indicating the presence of corresponding rickettsial antibodies in patient's serum. No agglutination indicated the absence of antibodies. This slide agglutination was done with doubling dilutions of each serum -1:80, 1:160 \& 1: 320. According to the baseline titer for rickettsial diseases reported previously, a single Weil-Felix titer of 1:160 or more was considered as diagnostically significant. ${ }^{15}$

Actually, this non-specific heterophil agglutination test is based on the principle that some strains of proteus share common somatic antigens with certain species of Rickettsiae. Therefore sera from patients of rickettsioses, containing rickettsial antibodies react with proteus antigen suspension \& thus produce visible agglutination. Usually antigen suspension of $\mathrm{P}$. vulgaris-OX2 react strongly with sera from patient infected with spotted fever group rickettsiae, except rocky mountain spotted fever (RMSF). Antigen suspension of $P$. vulgaris-OX19 reacts with sera of patient with typhus group as well as RMSF, while P. mirabilis-OXK antigen suspension strongly reacts with sera of patients with scrub typhus. $^{5}$

\section{Results}

Out of 453 serum samples, a total of 260 $(57.39 \%)$ showed significant seroreactivity (titer $\geq 1$ : 160) by Weil-Felix agglutination test. Among these 260 positive samples, 179 $(68.84 \%)$ sera showed reactivity only to one Weil-Felix antigen (single antigen reactivity), of which 101 (38.84\%) were reactive to OX2 antigen, suggestive of spotted fever group rickettsiae, 65 (25\%) were reactive to OXK antigen, suggestive of scrub typhus \& 13 (5\%) showed significant titers to OX19 antigen, indicating tick typhus or typhus fever group of rickettsiae. Remaining 81 (31.15\%) Weil-Felix positive samples showed significant titers to more than one Proteus antigen (mixed antigen reactivity), making it difficult to interpret the result. Analyzing all the mixed titers, it was evident that 32 samples (32/260; $12.30 \%$ ) were reactive to both OX2 \& OX19 antigens, 38 (38/260; 14.61\%) were reactive to both OXK \& OX2 antigens, 3 (3/260; $1.15 \%)$ were positive for both OX19 \& OXK antigens and $8(8 / 260 ; 3.07 \%)$ samples showed significant titers to all the three (OXK, OX2 \& OX19) antigens [Table 01]. 
Out of these 260 Weil-Felix positive cases, 118 (45.38\%) were male \& 142 (54.61\%) were female, indicating a female predominance [Figure 01]. Age analysis of the positive cases revealed that, maximum 95 (36.53\%) were in adolescent \& young adult age group $>15-30$ years, followed by 80 $(30.76 \%)$ cases in pediatric age group $0-15$ years. Middle aged group $>30-45$ accounted for 50 (19.23\% cases) \& elderly age group $>60$ years accounted for only $8(3.07 \%)$ cases [Figure 02]. This study also documented that, $148 / 260(56.92 \%)$ cases were from different rural areas of Mymensingh \& remaining $112 / 260(43.07 \%)$ were from urban locality, indicating increased occurrence of rickettsial illness among rural population [Table 02]. Month wise analysis of all the weil-Felix positive cases revealed that, higher number of the positive cases were documented during the months of August, September, October \& November \& thus accounted for $30(11.53 \%)$, 55 (21.15\%), 40 (15.38\%) \& $33(12.69 \%)$ cases respectively. It indicates that, incidence of rickettsial illness is more during the late rainy season \& the beginning of the winter months [Figure 03].

All the seropositive cases were presented with fever. Apart from fever, headache (145; $55.76 \%$ ) was the most common manifestation, followed by myalgia (132; 50.76\%) and cough (108; 41.53\%). Eschar was present only in 24 (9.23\%) cases and 27 (10.38\%) patients had rashes on their skin (Figure 04 showing eschar). 39 (15\%) cases were presented with anemia, $28(10.76 \%)$ cases had jaundice and only 11 (4.23\%) patients were presented with neck rigidity [Table 03].

Table 01: Distribution of seropositive cases according to Weil-Felix antigens $(n=260)$

\begin{tabular}{|c|c|c|c|c|}
\hline \multicolumn{2}{|c|}{ Weil-Felix antigens } & \multirow{2}{*}{$\frac{\text { No. of cases }}{101}$} & \multicolumn{2}{|c|}{ Percentage (\%) } \\
\hline \multirow{3}{*}{$\begin{array}{l}\text { Single } \\
\text { antigen }\end{array}$} & $\mathrm{O} 2$ & & 38.84 & \multirow{3}{*}{$\begin{array}{c}68.84 \% \\
(179 / 260)\end{array}$} \\
\hline & OXK & 65 & 25 & \\
\hline & OX19 & 13 & 5 & \\
\hline \multirow{4}{*}{$\begin{array}{l}\text { Mixed } \\
\text { antigens }\end{array}$} & $\mathrm{OXK}+\mathrm{OX} 2$ & 38 & 14.61 & \multirow{3}{*}{$\begin{array}{l}31.16 \% \\
(81 / 260)\end{array}$} \\
\hline & $0 \times 2+0 \times 19$ & 32 & 12.30 & \\
\hline & OXK+OX19 & 3 & 1.15 & \\
\hline & $\begin{array}{c}\text { OXK+OX2+ } \\
\text { OX19 }\end{array}$ & 08 & 3.07 & \\
\hline \multicolumn{2}{|c|}{ Total } & 260 & \multicolumn{2}{|c|}{100} \\
\hline
\end{tabular}

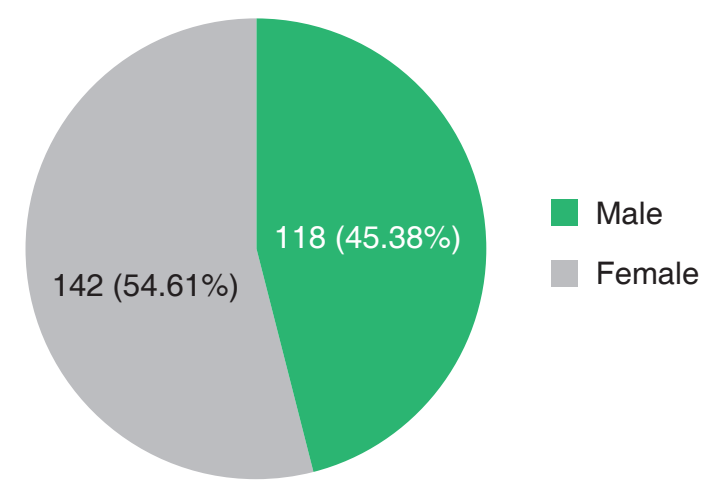

Figure 01: Sex distribution of Weil-Felix positive cases $(n=260)$ 


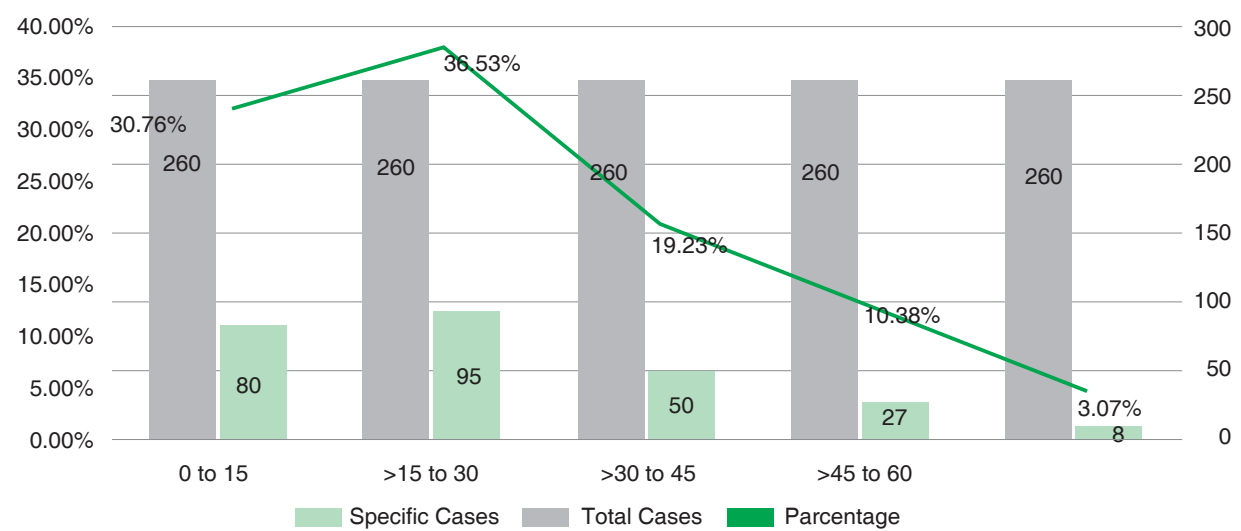

Figure 02: Age distribution of Weil-Felix positive cases $(n=260)$

Table 02: Distribution of Weil-Felix positive cases according to locality $(n=260)$

\begin{tabular}{ccc}
\hline Locality & No. of cases & percentage \\
\hline Urban & 148 & 56.92 \\
\hline Rural & 112 & 43.07 \\
\hline Total & 260 & 100 \\
\hline
\end{tabular}

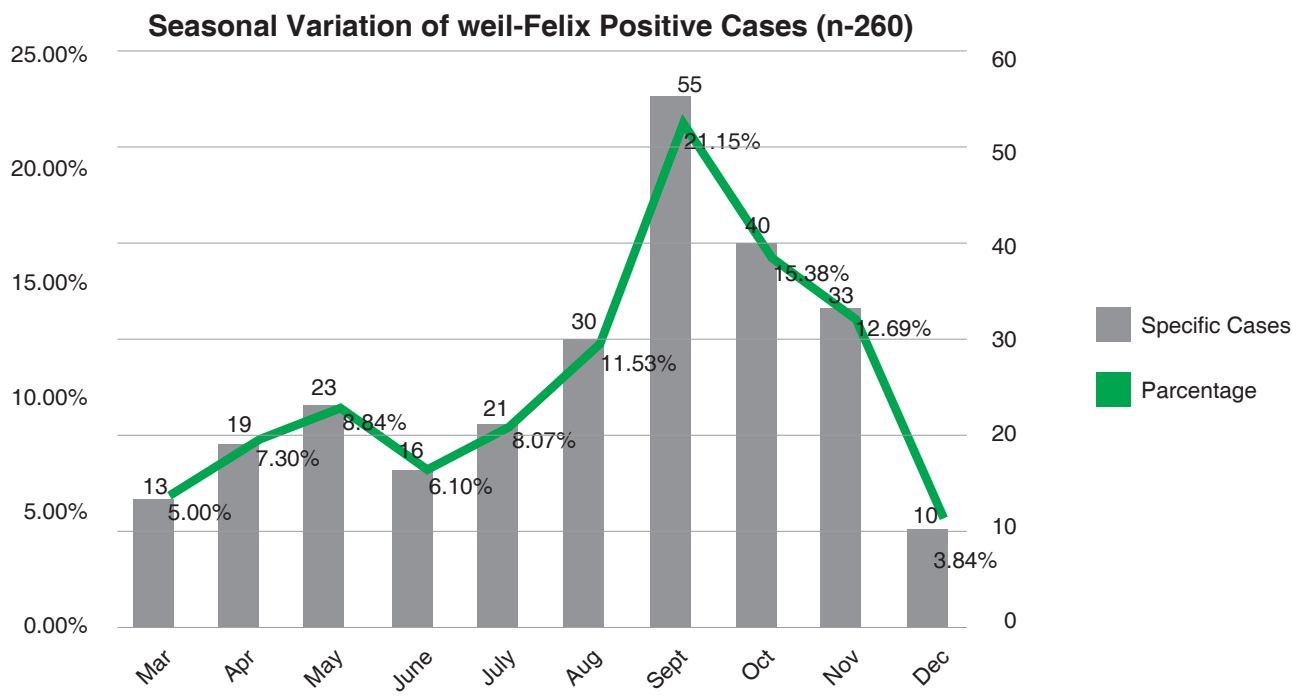

Figure 03: Month wise distribution of Weil-Felix positive cases $(n=260)$

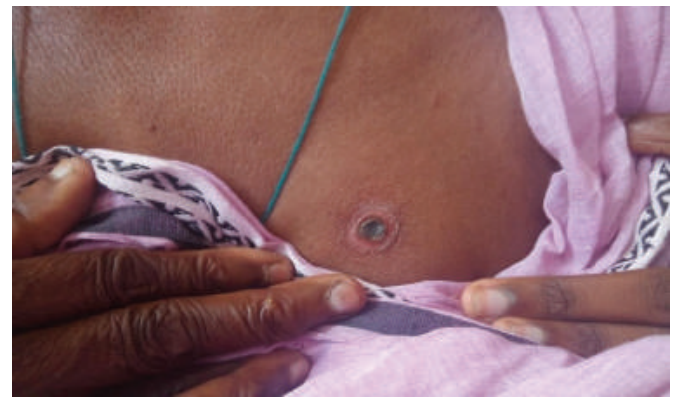

Figure 04: Eschar on the upper left chest of a female patient, documented during the present study. 
Table 03: Clinical presentations of Weil-Felix positive cases $(n=260)$

\begin{tabular}{lll}
\hline Clinical features & No. of cases & Percentage (\%) \\
\hline Fever & 260 & 100 \\
\hline Headache & 145 & 55.76 \\
Myalgia & 132 & 50.76 \\
\hline Cough & 108 & 41.53 \\
\hline Vomiting & 41 & 15.76 \\
Eschar & 24 & 9.23 \\
\hline Respiratory distress & 29 & 11.15 \\
\hline Anemia & 34 & 13.07 \\
\hline Skin Rash & 25 & 9.61 \\
\hline Oliguria & 19 & 7.30 \\
\hline Jaundice & 28 & 10.76 \\
\hline Neck rigidity & 9 & 3.46 \\
\hline Lymphadenopathy & 30 & 11.53 \\
\hline Splenomegaly & 18 & 6.81 \\
\hline Hepatomegaly & & 7.30 \\
\hline
\end{tabular}

\section{Discussion}

Rickettsioses are neglected, re-emerging infectious diseases throughout the world, which can be considered as a major cause of undifferentiated febrile illness in Asia-Pacific region, including several parts of indian-subcontinent. ${ }^{3,16-18}$ It is grossly under-diagnosed due to wide range of non-specific clinical features, low index of suspicion and lack of accurate diagnostic facilities, leading to significant morbidity \& mortality. $5,19,20$ So early \& accurate laboratory diagnosis of rickettsiosis is very essential to prevent potential life-threatening complications.

Although rickettsiae can be detected by laboratory culture, it is not routinely done as a diag- nostic tool, because culture is very cumbersome \& there is high chance of spreading infection to the laboratory personnel. Therefore, the mainstay of diagnosis of rickettsial illness depends on different serologic \& molecular techniques. Serology includes the gold standard-"microimmunofluorescence", followed by latex agglutination test, ELISA \& immunoperoxidase assay (IPA). ${ }^{5,21}$ But these specific serological tests are not readily available in many developing countries of South-East Asia. Moreover, they require highly trained personnel and standardization of antigens for those tests may vary among different laboratories leading to significant inconsistencies in results. ${ }^{5}$ Though molecular method like PCR for detection of rickettsial 
DNA is now available in many institutions, it is not realistic due to high cost issues \& lack of trained personnel. ${ }^{3}$ Thus in resource poor countries, the non-specific \& less sensitive Weil-Felix test still serves as a useful as well as affordable tool for diagnosis of rickettsiosis. Though it is not a standard test, it is still reasonably good when it is considered to evaluate a fever of unknown origin (FUO) \& thus can serve as an initial screening test to guide the physician for starting early treatment. ${ }^{2,11}$

Present study reported $57.40 \%$ (260/453) seropositivity for rickettsial infections by Weil-Felix agglutination test. A recent study among suspected rickettsial cases in Goa (India), reported 58.18\% (32 / 55) seropositivity \& a hospital based study in Bengaluru (India), also demonstrated 59.22\% (61/103) seropositivity for rickettsial illness by Weil-Felix test. ${ }^{22,23}$ These results were very much consistent with present study.

Analyzing the reactivity of Weil-Felix antigen in present study, it was evident that $38.84 \%$ $(101 / 260)$ sera were reactive to only OX2, suggestive of spotted fever group rickettsiae. $25 \%(65 / 260)$ sera were reactive to only OXK \& 5\% (13/260) were positive for only OX19 indicating scrub typhus \& typhus group of rickettsiae respectively. A study on serological diagnosis of rickettsial iillness in Delhi (India) reported $39.94 \%$ positivity for OX2, $42.6 \%$ positivity for OXK \& 8.1\% reactivity for OX19 antigen. ${ }^{15}$ A previous study in Mymensingh, Bangladesh showed $50 \%$ sera were positive for OX2, $12 \%$ were for OXK \& 5.33\% were reactive for OX19. ${ }^{24}$ These results were more or less similar to present findings. Present study also documented that among the seropositive cases, $31.15 \%(81 / 260)$ sera were reactive to more than one antigen (mixed antigen), of which maximum $14.61 \%$ (38/260) showed reactivity to both OXK \& OX2 antigens, followed by $12.30 \%$ (32/260) sera to both OX2 \& OX19. A previous study in Parel (India) documented nearly very similar result, where $41.44 \%$ sera showed mixed seropositivity, of which $14.43 \%$ were reactive for both OXK \& OX2 antigens. ${ }^{1}$
Among 260 seropositive cases, this study documented 118 (45.38\%) were male \& 142 $(54.61 \%)$ were female, indicating a female preponderance. A similar study on Weil-Felix test to detect rickettsial infection in Maharashtra (India) reported more number of female patients $(66.66 \% ; 18 / 27)$ than male $(33.33 \%$; 09/27), among the total seropositive samples. $^{12}$ A study during an outbreak of scrub typhus in Pondicherry (India), also reported the occurrence of increased number of female cases $(28 / 50 ; 56 \%)$ than male $(22 / 50$; $44 \%) .{ }^{20}$ These reports were similar enough to present study. In contrast to this findings, a study on serological evidence of rickettsial infection in Karnataka (India), reported increased number of seropositivity among male $(57.14 \%)$ subjects than female $(42.86 \%) .{ }^{5}$ Slight gender variations of rickettsial cases according to different studies are most likely due to occupational pattern and differing social role of men and women in different study population. ${ }^{25}$

This study documented that, most of the seropositive cases $(95 / 260 ; 36.53 \%)$ were in adolescent \& young adult age group ( $>15-30$ years), followed by 80 (30.76\%) cases in pediatric age group (0-15 years). Minimum number of seropositive cases $(8 / 260 ; 3.07)$ were evident in elderly age group ( $>60$ years). Previous study in Mymensingh (Bangladesh), reported maximum $40.58 \%$ cases were in age group $>15-30$ years. A study in Karnataka (India) showed $35.71 \%$ (5/14) cases were in age group $>15-30$ with no cases documented in elderly age group $>60$ years. ${ }^{5}$ Another study in Bengaluru (India), documented $27.58 \%(8 / 29)$ cases were in pediatric age group 0-15 years. ${ }^{3}$ All these reports were consistent with present findings. Intense outdoor and field exposure due to occupational or recreational activity, may be the possible reason of more rickettsial cases among this active adolescent \& young adult population (>15-30years), because outfield activity makes them vulnerable to encounter vector mites \& ticks. ${ }^{10}$ 
Present study reported more number of cases (148/260; 56.92\%) were from different rural areas of Mymensingh region. A study in Maharashtra (India) also documented that, rickettsial fever was more prevalent in rural areas of western Maharashtra. ${ }^{7}$ Rural people are at more risk for acquisition of rickettsial illness, as because they are more likely to remain in contact with reservoir animals (wild \& farm) than urban population. ${ }^{26}$ Studies from China also suggest that, a closer proximity of a person's home to the paddy field, scrub vegetation, ditches or wood piles within a rural village areas may increase the risk of scrub typhus, due to more chance of encountering the vector mites, ${ }^{25}$ which may also explain the increased occurrence of rickettsiosis in rural population. Present study found that most of the seropositive cases were clustered from month of August to November, of which September accounted for maximum number of cases $(55 / 260 ; 21.15 \%)$, followed by October $(40 / 260 ; 15.38 \%)$. This period is actually the end of rainy season (post-monsoon) \& a study on rickettsial illness in Karnataka (India) also reported increased number of seropositive cases were evident during this late rainy season (August to November). The increased incidence of rickettsial illness particularly scrub typhus at the end of the rainy season (post-monsoon) is expected as rich growth of secondary or transitional vegetations favours the growth of larval trombiculid mites, leading to increased man-vector contact. ${ }^{27}$

All the documented seropositive cases of this study had fever. Apart from fever, headache $(145 ; 55.76 \%)$ was the most common presenting problems, followed by myalgia (132; $50.76 \%)$ \& cough $(108 ; 41.53 \%)$. Nearly similar result was documented in a previous study in Mymensingh, where headache (42.02\%) was the most common manifestation, followed by myalgia $(24.63 \%)$ \& cough. ${ }^{24}$ This study showed skin rashes \& eschar were present in $10.38 \%(27 / 260) \& 9.23 \%(24 / 260)$ cases respectively. A study in Pondicherry (India), also documented $20 \%$ (13/65) scrub typhus cases with eschar. ${ }^{28}$ This study also found anemia (39; 15\%), jaundice (28; 10.76\%), vomiting $(48 ; 18.46 \%)$ as presenting problems, which is consistent with the findings of Mahajan et al., from India. ${ }^{29}$ We have documented oliguria in 19 (7.30\%) cases indicating acute kidney injury (AKI). This finding is supported by a hospital based study in Chandigarh (India), which reported 26.53\% (13/49) of scrub typhus cases having oliguria and they were all diagnosed to have $\mathrm{AKI} .^{30}$ We have also documented 11 (4.23\%) seropositive cases with neck rigidity, which is a definitive sign of meningitis. $26 \%$ (17/65) scrub typhus cases with evidence of neck rigidity were reported from a hospital based study in Pondicherry, India. ${ }^{28}$ So, rickettsial illnesses, particularly scrub typhus should be regarded as an emerging cause of meningitis in clinical practices. This study also recorded that hepatomegaly \& splenomegaly were found in 19 $(7.30 \%) \& 18(6.81 \%)$ cases respectively. Two separate studies on rickettsial illness in Karnataka \& Bengaluru (India), also documented a good number of seropositive cases with hepatomegaly \& splenomegaly. ${ }^{3,11}$ From the above discussion, it is very clear that rickettsial illness usually manifests as a non-specific febrile illness, but disease spectrum can be extended into fatal multi-organ involvement also. ${ }^{31}$

So, this study clearly gives us an insight of the febrile patients with suspected rickettsiosis presenting to a tertiary care hospital \& it is obvious that there are lots of genuine rickettsial cases, usually presenting with self-limiting non-specific manifestations as well as with multi-organ involvement like-hepatosplenomegaly, acute renal failure, hepatic failure, meningoencephalitis etc. So awareness among physicians \& early diagnosis is very essential to prevent morbidity \& mortality.

\section{Limitation}

Main limitation of the study was that the seropositive cases by Weil-Felix test, could not be compared with ELISA, immunofluorescence or other confirmatory tests in reference laboratories. 


\section{Conclusion}

Our results show that, rickettsial infections are prevalent in Mymensingh region of Bangladesh \& it should be considered as one of the most important causes of Fever of Unknown Origin. Nationwide active surveillance of rickettsial diseases is required to know the actual burden of the disease. In spite of all the drawbacks associated with Weil-Felix test, in a resource poor country like Bangladesh, it still serves as the available tool for diagnosis of the rickettsial illness to guide the clinicians for establishing early treatment. With proper use of standardized antigens, inclusion of positive serum controls \& proper clinical correlation with patient's sign-symptoms, Weil-Felix test can be considered as a cost effective option for initial screening of rickettsial illness.

\section{References}

1. Sanap SS, Thakur VA, Maniar JM, Vasave SV and Vaidya SP. Weil-Felix Test - A Diagnostic Tool for Rickettsial Diseases. Austin J Clin Pathol. 2017; 4(1): 1046. ISSN:2381-9170

2. Sudhindra KS, Sumanta A, Shubha DS, Narayana Murthy C, Srinivasa Setty TK. Weil Felix test for detection of Rickettsial infections. Indian $J$ Microbiol Res 2017;4(2):216-219

3. Rashmi KS, Murthy NS, Ravikumar KL. Rickettsial Diseases: A Study Evidenced by Weil-Felix Test in a Tertiary Care Hospital. Int J Sci Stud 2015;3(8):128-131

4. Mahajan, Sanjay. Ricketsial diseases. The Journal of the Association of Physicians of India. 2012; 60. 37-44.

5. Raghu Kumar K, Tejashree $\mathrm{P}$, Basavarajappa K. Serological evidence of rickettsial infections in and around Davangere. International Journal of Medical Science and Public Health. 2015; 4: 1731-1734.

6. Oberoi A, Singh N. Rickettsiae Infections-Classification. JK Science.
2010; 12(2) : 57-59.

7. Nigwekar p, kavar Y, Shrikhande DY, Ashok KC. Clinicopathological profile of Rickettsial fever in a rural area of western Maharashtra, India. Parvara Med Rev. 2013; 5(3)

8. Rungta N. Scrub typhus: Emerging cause of multiorgan dysfunction. Indian journal of critical care medicine: peer-reviewed, official publication of Indian Society of Critical Care Medicine. 2014; 18(8) : 489-491

9. Nawab T, Srinivasa S, Reddy SP. A clinical study of rickettsial disease and its manifestations. Current Pediatric Research. 2015; 19.

10. Rahi M, Gupte MD, Bhargava A, Varghese GM, Arora R. DHR-ICMR Guidelines for Diagnosis and Management of Rickettsial Disease in India. Indian Journal of Medical Research. 2015; 141(4): 417-422.

11. Udayan U, Dias M, Machado S. A hospital based study of Rickettsial diseases evidenced by Weil Felix test in a tertiary care hospital. CHRISMED J Health Res. 2014;1:150-3

12. Danave D, Kothadia S. Role of Weil Felix Test for Rickettsial Infections. IOSR Journal of Dental and Medical Sciences (IOSR-JDMS). 2015; 14(7): 52-54.

13. Sood A, Chauhan1S. Rickettsial Diseases: An Urgent Need to Upgrade Diagnostic Facilities. International Journal of Recent Trends in Science and Technology. 2013; 7: 20-21

14. Kingston HW, Hossain M, Leopold S, Anantatat T, Tanganuchitcharnchai A, Sinha I, Plewes K, Maude, RJ, Chowdhury, MH, Paul S, Uddin RAME. Rickettsial illnesses as important causes of febrile illness in Chittagong, Bangladesh. Emerging infectious diseases. 2018; 24(4): 638-645.

15. Mittal V, Gupta N, Bhattacharya D, Kumar K, Ichhpujani R, Singh S, et al. Serological evidence of rickettsial infections in Delhi. The Indian journal of 
16. Azad AF, Beard CB. Rickettsial pathogens and their arthropod vectors. Emerging infectious diseases. 1998; 4(2): 179-186.

17. World Health Organization. Global surveillance of rickettsial diseases: Memorandum from a WHO meeting. Bull WHO. 1993; $71:$ 293-296.

18. Mahajan SK, Bakshi D. Acute reversible hearing loss in scrub typhus. J Assoc Physicians India. 2007 Jul; 55:512-4. PMID: 17907503.

19. Maude RR, Maude RJ, Ghose A, Amin, MR, Islam MB, Ali M, Bari MS, Majumder MI, Tanganuchitcharnchai A, Dondorp AM, Paris DH. Serosurveillance of Orientia tsutsugamushi and Rickettsia typhi in Bangladesh. The American journal of tropical medicine and hygiene. 2014; 91(3): 580-583.

20. Vivekanandan M, Mani A, Priya YS, Singh AP, Jayakumar S, Purty $S$. Outbreak of scrub typhus in Pondicherry. J Assoc Physicians India. 2010; 58(1) : 24-28.

21. Rathi N, Rathi A. Rickettsial infections: Indian perspective. Indian pediatrics. 2010; 47: 157-16

22. Narvencar K, Kaur G, Rodrigues S. Rickettsial Infections in Goa-Not Just Scrub Typhus!. Journal of The Association of Physicians of India. 2017; 65: 24-27.

23. Kalal BS, Puranik P, Nagaraj S, Rego S, Shet, A. Scrub typhus and spotted fever among hospitalised children in South India: Clinical profile and serological epidemiology. Indian journal of medical microbiology. 2016; 3(34): 293-298.
24. Ferdouse F, Hossain MA, Paul SK, Ahmed S, Mahmud MC, Ahmed R, et al. Rickettsia felis Infection among humans, Bangladesh, 2012-2013. Emerg Infect Dis $2015 ; 21: 1483-5$.

25. Trowbridge P, Premkumar PS, Varghese GM. Prevalence and risk factors for scrub typhus in South India. Tropical Medicine \& International Health. 2017; 22(5):.576-582.

26. Lledó L, Domínguez-Peñafiel $\mathrm{G}$, Giménez-Pardo C, Gegúndez I, González R, Saz JV. Molecular and serological study of rickettsial infection in humans, and in wild and farm animals, in the province of Burgos, Spain. Vector Borne Zoonotic Dis. 2014; 14(6):383-388.

27. Anitha PK, Hoti SL, Kanungo R, Jambulingam $P$, Nazeer $Y$. Occurrence of Orientia Tsutsugamushi Genotypes in Areas of Union Territory of Puducherry and Tamil Nadu State, India. J Emerg Infect Dis. 2017; 2(124): e1000124.

28. Viswanathan $\mathrm{S}$, Muthu V, Iqbal $\mathrm{N}$, Remalayam B, George T. Scrub typhus meningitis in South India-a retrospective study. PLoS one. 2013; 8(6): e66595.

29. Mahajan S, Kashyap R, Kanga A, Sharma V, Prasher B, Pal L. Relevance of Weil-Felix test in diagnosis of scrub typhus in India. JAPI. 2006; 54: 619

30. Kumar V, Kumar V, Yadav AK. Scrub typhus is an under-recognized cause of acute febrile illness with acute kidney injury in India. PLoS Negl Trop Dis. 2014; 8(1): e2605.

31. Jeong YJ, Kim S, Wook YD, Lee JW, Kim KI, Lee SH. Scrub typhus: clinical, pathologic, and imaging findings. Radiographics. 2007; 27(1) : 161-172. 